\title{
Evacuation of rectilinear polygons ${ }^{\star}$
}

\author{
Sándor Fekete, Chris Gray, and Alexander Kröller \\ Department of Computer Science, TU Braunschweig, Germany. \\ \{fekete,gray, kroeller\}@ibr.cs.tu-bs.de
}

\begin{abstract}
We investigate the problem of creating fast evacuation plans for buildings that are modeled as grid polygons, possibly containing exponentially many cells. We study this problem in two contexts: the "confluent" context in which the routes to exits remain fixed over time, and the "non-confluent" context in which routes may change. Confluent evacuation plans are simpler to carry out, as they allocate contiguous regions to exits; non-confluent allocation can possibly create faster evacuation plans. We give results on the hardness of creating the evacuation plans and strongly polynomial algorithms for finding confluent evacuation plans when the building has two exits. We also give a pseudopolynomial time algorithm for non-confluent evacuation plans. Finally, we show that the worst-case bound between confluent and non-confluent plans is $2-O\left(\frac{1}{k}\right)$.
\end{abstract}

\section{Introduction}

A proper evacuation plan is an important requirement for the health and safety of all people inside a building. When we optimize evacuation plans, our goal is to allow people to exit the building as quickly as possible. In the best case, each of a building's exits would serve an equal number of the building's inhabitants. However, there might be cases in which this can not happen. For instance, when there is a bottleneck between two exits, it might make sense for most of the building's inhabitants to stay on the side of the bottleneck closer to where they begin, even if this means that one exit is used more than the other.

In this research, we study the computation of evacuation plans for buildings that are modeled as grid polygons. We make the assumption that every grid square is occupied by exactly one person and that at most one person can occupy a grid square at any given time. The first assumption may seem a bit contrived, but in many cases it is impossible for a building designer to know exactly where people will be in the building in the moments before an evacuation, and this pessimistic view of the situation is the only sensible one to take. Also remember that in some cases, such as in airplanes, the situation in which nearly every bit of floor space is occupied before an evacuation is more common than the alternative.

\footnotetext{
* This research was funded by the German Ministry for Education and Research (BMBF) under grant number 03NAPI4 "ADVEST", which fully funded Chris Gray.
} 
Evacuation plans can be divided into two distinct types. In the first, signs are posted that direct every person passing them to a specific exit. In the second, every person is assigned to a distinct exit that does not necessarily depend on the exits to which his or her neighbors are assigned. The first type of evacuation plan generates what is known as a confluent flow and the second generates a non-confluent flow. More precise definitions of these terms will be given later.

It is clear that in a non-confluent flow, people can be evacuated as quickly as in a confluent flow, and we show that in certain instances people can be evacuated significantly more quickly in a non-confluent flow than in a confluent flow. However, a confluent flow is significantly easier to carry out than a nonconfluent one, so we also give results related to it. We first show that the problem of finding an optimal confluent flow belongs to the class of NP-complete problems if the polygon has "holes"- that is, if it represents a building with completely enclosed rooms or other spaces. We then give an algorithm with a running time linear in the description complexity of the region (which can be exponentially smaller than the number of cells) that computes an evacuation plan for buildings without holes that have two exits; a generalization to a constant number of exits is more complicated, but seems plausible. Finally, we show that the worst-case ratio between the evacuation times for confluent flows and non-confluent flows for $k$ exits is $2-O\left(\frac{1}{k}\right)$.

\section{$1.1 \quad$ Preliminaries}

We are given a rectilinear polygon $P$ on a grid. There exists, on the boundary of $P$, a number of special grid squares known as exits. We call the set of exits $\mathcal{E}=\left\{e_{1}, \ldots, e_{k}\right\}$. We assume that every grid square in $P$ contains a person. A person can move vertically or horizontally into an empty grid square or an exit. The goal is to get each person to an exit as quickly as possible. When an exit borders more than one grid square, we specify the squares from which people can move into the exit.

The area of $P$ is denoted by $A$ and the number of vertices of $P$ is denoted by $n$. Note that $A$ can be exponential in $n$. The set of people that leave $P$ through the exit $e$ is called the $e$-exit class. We also write exit class to refer to the set of people who leave through an unspecified exit. The grid squares that are adjacent to the boundary of $P$ are known as boundary squares.

There are two versions of the problem that we consider. We call these confluent flows and non-confluent flows. In the first, we add the restriction that every grid square has a unique successor. Thus, for every grid square $s$, people passing through $s$ leave $s$ in only one direction. This restriction implies that evacuation plans are determined by space only. It does not exist for non-confluent flows. It can be argued that informing people which exit to use is easier in the case of confluent flows since a sign can be placed in every grid square, informing the people who pass through it which exit to use. However, we show that non-confluent flows can lead to significantly faster evacuations.

The major difficulty in the problem comes from bottlenecks. We define a $k$ bottleneck to be a rectangular subpolygon $B$ of $P$ such that two parallel boundary 
edges of $B$ are the same as two edges of $P$, where the distance between the common edges is $k$.

\section{$1.2 \quad$ Related Work}

The problem when restricted to confluent flows is similar in many ways to the unweighted Bounded Connected Partition (or 1-BCP) problem [12]. In this problem, one is given an unweighted, undirected graph $G=(V, E)$ and $k$ distinguished vertices. The goal is to find $k$ connected subsets of $V$, where each subset contains exactly one of the distinguished vertices and where each has the same cardinality. If we define the dual of the grid contained in our polygon to be the graph formed by connecting adjacent faces and let the distinguished vertices be the exits, then 1-BCP is clearly the same as evacuation restricted to confluent flows with $k$ exits.

It was independently shown by Lovász [8] and Györi [7] that a solution to the 1 -BCP problem can be found for every graph that is $k$-connected. However, their proofs are not algorithmic. Thus, there has been some work on finding partitions of size $k$ for low values of $k$. For example, Suzuki et al. give an algorithm for 2partitioning 2-connected graphs [14]. One algorithm claiming a general solution for $k$-partitioning $k$-connected graphs [10] is incorrect.

Unfortunately, when $P$ contains 1-bottlenecks, the graph obtained by finding the dual of the grid inside $P$ is 1-connected. Therefore, the results of Lovás and Györi do not apply. Also, since the dual of the grid contained in our polygon can have size exponential in the complexity of the polygon, we would probably need to merge nodes and then assign weights to the nodes of the newly-constructed graph. The addition of weights, however, makes the BCP problem NP-complete, even in the restricted case in which the graph is a grid [3].

Another connection is to the problem of partitioning polygons into subpolygons that all have equal area. The confluent version of our problem, indeed, can be seen as the "discrete" version of that problem. The continuous version has been studied. One interesting result from this study is that finding such a decomposition while minimizing the lengths of the segments that do the partitioning is NP-hard even when the polygons are orthogonal [1]. However, polynomial algorithms exist for the continuous case when that restriction is removed [9].

Baumann and Skutella [2] consider evacuation problems modeled as earliestarrival flows with multiple sources. They achieved a strongly-polynomial-time algorithm by showing that the function representing the number of people evacuated by a given time is submodular. Such a function can be optimized using the parametric search technique of Megiddo. Their approach is different from ours in that they are given an explicit representation of the flow network as input. We are not given this, and computing the flow network that is implicit in our input can take exponential time. Also, their algorithm takes polynomial time in the sum of the input and output sizes. However, the complexity of the output can be exponential in the input size.

Another, perhaps more surprising, related problem is machine scheduling. Viewed simply, confluent flows correspond to non-preemptive scheduling prob- 
lems, while non-confluent flows correspond to preemptive scheduling problems. The NP-hardness result for optimal confluent flows is inspired by the hardness of scheduling jobs on non-preemptive machines [5], while the worst-case ratio between confluent and non-confluent flows is inspired by the list-scheduling approximation ratio [6].

\section{Confluent Flows}

As mentioned in the introduction, in a confluent flow, every grid square has the property that all people that pass through it use the same exit.

In this section, we present our results related to confluent flows. First, we show the NP-completeness of the problem of finding an optimal evacuation plan with confluent flows in a polygon with holes. This holds even for polygons with two exits. We then give a linear-time algorithm for polygons with two exits.

\subsection{Hardness}

Weak NP-hardness with two exits. We first show that the evacuation problem with confluent flows is NP-hard if we allow $P$ to have holes. We reduce from the problem PARTiTiOn, which is well-known to be NP-complete [5]. In this problem, we are given a set $S=\left\{c_{1}, c_{2}, \ldots, c_{m}\right\}$ of integers and we are asked to determine whether we can find $S_{1}, S_{2} \subseteq S$ such that $\sum_{c_{h} \in S_{1}} c_{h}=\sum_{c_{i} \in S_{2}} c_{i}$.

We note that if we scale all of the numbers in a PARTITION instance by an integer $\ell$, the answer remains the same - that is, a partition can be found in the new set if and only if a partition could be found in the old set - but the difference between the size of non-optimal sets is at least $\ell$. This is because

$$
\sum_{c_{h} \in S_{1}} \ell c_{h}-\sum_{c_{i} \in S_{2}} \ell c_{i}=\ell\left(\sum_{c_{h} \in S_{1}} c_{h}-\sum_{c_{i} \in S_{2}} c_{i}\right) \geq \ell
$$

if the sums are not equal.

To transform the PARTITION problem into our problem, we first scale the input by a factor of $2 m+1$. We then do the following to make the polygon $P$ :

- We make a rectangle whose width is $m+1+\sum_{c_{i} \in S} c_{i}$ and whose height is 5 .

- We remove all the grid squares of $P$ on the second and fourth rows except those that are at position $\sum_{i=1}^{j} c_{i}+j$ for all $0<j \leq m$.

- We remove all grid squares from the third row that are at position $\sum_{i=1}^{j} c_{i}+j$ for all $0<j \leq m$.

- We add a large number of squares (at least equal to the current area of $P$ ) to the left end of the first and fifth rows.

- We add an exit $e_{1}$ to the right end of the first row and an exit $e_{2}$ to the right end of the fifth row. 


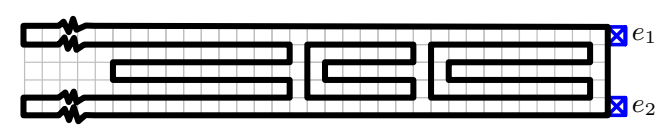

Fig. 1. The polygon $P$ given a PARTition instance of $\{11,6,9\}$. To keep the picture a manageable size, the elements have not been scaled and the left ends of the first and fifth rows are truncated.

See Figure 1 for a small example. We say that the connected sets of grid squares in the third row each correspond to one of the elements of the given PARTITION instance. This leads to the following lemma.

Lemma 1. The polygon $P$ with holes can be divided into two confluent exit classes of equal size if and only if the given instance of PARTITION has a "yes" answer.

Proof. First, assume that the PARTition instance has a "yes" answer and that we are given $S_{1}$ and $S_{2}$, the sum of whose elements are equal. We then take the grid squares from the third row that correspond to elements in the set $S_{1}$ and send them to $e_{1}$. We take the remaining grid squares from the third row and send them to $e_{2}$. We also send all the grid squares in the first and second rows to $e_{1}$ and all the grid squares in the fourth and fifth rows to $e_{2}$.

It is obvious that the number of grid squares in the rows other than the third row are equal. Since the sum of the elements in $S_{1}$ is equal to the sum of the elements in $S_{2}$, the number of grid squares sent to $e_{1}$ and $e_{2}$ is equal.

Now assume that the PARTITION instance does not have a "yes" answer. This implies that $\left|\sum_{c_{i} \in S_{1}} c_{i}-\sum_{c_{j} \in S_{2}} c_{j}\right| \geq 2 m+1$ for any $S_{1}$ and $S_{2}$ that are subsets of $S$. Since we are dealing with a confluent flow, if any grid square from the first row exits through $e_{2}$, then all grid squares to the left of it must exit through $e_{2}$. This would mean that the number of grid squares exiting through $e_{2}$ would be more than $2 / 3$ the number of grid squares in $P$ (because of the "large number" of grid squares added to the left end of the first and fifth rows). Therefore, we can assume that no grid square from the first row exits through $e_{2}$ and similarly we can assume that no grid square from the fifth row exits through $e_{1}$. This implies that the only grid squares that can "choose" which exit to go through are those in the second, third, and fourth rows.

There are exactly $2 m$ grid squares in the second and fourth rows. Because the PARTITION instance does not have a "yes" answer, the number of grid squares from the third row going through $e_{1}$ has a difference of at least $2 m+1$ with the number of grid squares going through $e_{2}$. Therefore, there is no way that the number of grid squares going through $e_{1}$ is equal to the number of grid squares going through $e_{2}$.

We define the decision version of the problem of evacuation with confluent flows to be: "Given a grid polygon $P$ with $k$ exits and a natural number $\ell$, can a confluent flow be found in which the largest exit class has size at most $\ell$ ?" 
Since areas of polygons can be computed in time proportional to their number of vertices, we can verify if a solution is correct in $O(k n)$ time (where $n$ is the number of vertices of the polygon and $k$ is the number of exits). This, along with Lemma 1, implies that the decision problem is NP-complete in polygons with holes. We summarize this result in the following theorem.

Theorem 1. The problem of finding an optimal confluent flow in a polygon with holes is NP-complete.

Strong NP-hardness. Theorem 1 shows that the problem is weakly NP-complete. This means that the hardness of the problem depends on the areas of subpolygons being exponential in the complexity of the input. This implies that a pseudopolynomial algorithm might exist.

However, we will now show that if we allow $O(n)$ exits, the problem is strongly $N P$-complete. This means that the problem is still NP-complete when all of its numerical parameters are polynomially bounded in the size of the input.

Our reduction is from Cubic Planar Monotone 1-in-3 Satisfiability (or CPM 1-IN-3 SAT for short). This is a variant of the SATISFIABILITY problem in which

- every clause contains exactly 3 literals,

- every variable is in exactly 3 clauses,

- the graph generated by the connections of variables to clauses is planar,

- every literal in every clause is non-negated, and

- a clause is satisfied if exactly one of its variables is true.

We use a reduction that is almost equivalent to the one showing that tiling a finite subset of the plane with right trominoes is NP-complete [11], so we will summarize that reduction and describe our modifications to it.

A right tromino is a type of tile that consists of only three grid squares arranged in an "L" shape. Moore and Robson showed [11] that tiling a finite subset of the plane (that is, a polygon with holes) with right trominoes is NPcomplete. Since their reduction contains no numerical parameters and all the coordinates used are polynomially-sized integers, it is clear that the problem is strongly NP-complete.

The reduction uses gadgets - small sections of polygons that can be put together to form a larger polygon - to create a polygon that can be tiled by right trominoes if and only if a given instance of CPM 1-IN-3 SAT is satisfiable. The reduction is as follows. First, the graph $G=(V, E)$ generated by connecting variables to clauses is embedded on a grid. The embedding must have the property that every vertex is on a grid point and the set of edges is a set of disjoint paths along grid lines. Then every grid point that is occupied is replaced by an appropriate gadget (rotated appropriately). The vertices that correspond to variables are replaced by variable gadget; those that correspond to clauses are replaced by a clause gadget. A grid point that is occupied by an edge is replaced by a wire gadget when the edge continues in the same direction through the grid 


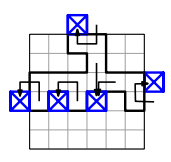

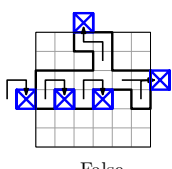

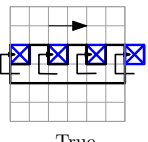

True

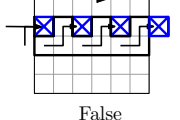

(b)

Fig. 2. (a) The variable gadget. (b) The wire gadget.

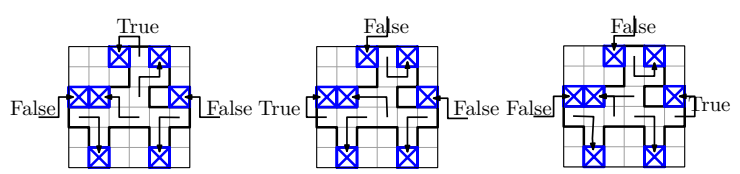

Fig. 3. The clause gadget, showing the three ways that people can evacuate such that each exit handles exactly three people.

point and by a turn gadget when the edge turns at the grid point. Since $G$ is a planar graph, it should be clear that the constructed polygon has holes.

The gadgets shown in Figures 2-4 are the same as those in the proof by Moore and Robson, we have only added exits to them so that the entire polygon can be evacuated with a maximum of three people going through each exit if and only if the polygon can be tiled by right trominoes.

The paths indicated in Figures 2-4 indicate the possible evacuation routes that satisfy the requirement that every exit handles at most three people. They correspond to the possible tiling of each gadget by right trominoes in the proof by Moore and Robson. We summarize.

Theorem 2. The problem of finding an optimal confluent flow in a polygon with holes and $O(n)$ exits is strongly NP-complete.

Since this implies that finding an optimal confluent flow in a polygon with holes in polynomial time is unlikely, we assume in the rest of the paper that $P$ is a simple grid polygon without holes.

\section{$2.2 \quad$ Two Exits}

When the polygon $P$ has only two exits, $e_{1}$ and $e_{2}$, we can find an optimal confluent flow in $O(n)$ time. We first present an algorithm that takes cubic time that can be modified fairly easily into an algorithm that takes linear time.

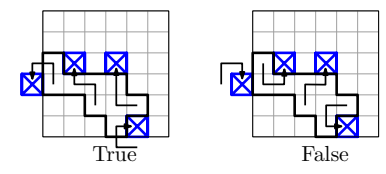

Fig. 4. The turn gadget. 
Naïve algorithm Notice that the case in which $P$ has two exits is simpler than the case in which $P$ has more exits because of the fact that both exit classes must each have one contiguous connection to the boundary of $P$.

We begin with a decomposition of $P$ into rectangles. This decomposition is the overlay of two simpler decompositions: the vertical and horizontal decompositions. The vertical decomposition of a rectilinear polygon $P$ is the partition of $P$ into rectangles by the addition of only vertical line segments. Similarly, the horizontal decomposition of $P$ is the partition of $P$ into rectangles by the addition of only horizontal line segments. We call the overlay $\omega$ and its dual graph $\omega^{*}$. We add the vertical and horizontal line segments from the grid points on opposite sides of $e_{1}$ and $e_{2}$ to $\omega$ as well. We then do the following for every pair of rectangles $r_{1}$ and $r_{2}$ in $\omega$ that have at least one edge of the boundary of $P$. We first ensure that $e_{1}$ is between $r_{1}$ and $r_{2}$ and that $e_{2}$ is between $r_{2}$ and $r_{1}$. We also ensure that there are no bottlenecks of size 1 between $r_{1}$ and $r_{2}$. If either of these conditions are not met, we proceed to the next pair of rectangles. We then set the $e_{1}$-exit class to be all the grid squares along the boundary of $P$ between $r_{1}$ and $r_{2}$. We then add all the grid squares surrounded by the $e_{1}$-exit class to the $e_{1}$-exit class. We call the area of the $e_{1}$-exit class $A_{1}$. We define the $e_{2}$-exit class similarly and call its area $A_{2}$. We call the larger of the two areas $A_{\ell}$ and its corresponding exit $e_{\ell}$. If $A_{\ell}$ is less than $A / 2$, we can divide the rest of the grid squares evenly among the exit classes - see Lemma 3 for details - and return the solution. Otherwise, we attempt to make the $e_{\ell}$-exit class as small as possible (while staying above $A / 2$ ) inside $r_{1}$ and $r_{2}$ and assign the rest of the grid squares to the other exit class. We maintain a variable that tracks the area of the smallest such exit class $e_{m i n}$. If we get through all the possible pairs $r_{1}$ and $r_{2}$ without finding a pair that we can return, we return $e_{\min }$.

Lemma 2. The algorithm presented above is correct and takes $O\left(n^{3}\right)$ time.

Proof. We assume that the algorithm presented in Section 2.3 is correct and runs in $O(n)$ time.

The time complexity of the algorithm comes from the fact that there are $O(n)$ rectangles of $\omega$ with at least one edge on the boundary of the polygon. We loop through each pair of these rectangles, and in each loop there is the possibility that we must find the area of an exit class.

To show that the algorithm is correct, we must argue that it finds the $e_{1}$ and $e_{2}$-exit classes and that

- both classes are connected and

- the size of the larger class is as small as possible.

Clearly, if we find connected exit classes that both have size $A / 2$ (which we do if we return early), we have satisfied both requirements. We find such a solution if it exists because we try all combinatorially unique starting and ending points for the connection of the $e_{1}$-exit class to the boundary of $P$.

Therefore, it remains to argue that we find the optimum solution for the case in which one exit class is larger than $A / 2$. In this case, we also try all 
combinatorially unique starting and ending points that minimize the size of both the $e_{1}$ and $e_{2}$ exit classes. The exit classes that we return consist of the smallest exit class that is larger than $A / 2$ and its complement (which must be smaller). This proves the lemma.

Linear algorithm The algorithm above has two steps that lead to it taking cubic time: the loop over all pairs of rectangles on the boundary of $P$ and the computation of the minimum area for an exit class that has a connection to the boundary that begins in one of the rectangles and ends in the other. In this section, we give a more clever solution that avoids these problems.

We begin by observing that if we update the area, each time we change the starting and ending points of the connection of the $e_{1}$-exit class to the boundary of the polygon rather than computing it anew, the total time spent computing the area depends on the sum of the complexities of the updated areas.

We also observe that we loop over the rectangles of $\omega$ with at least one edge attached to the boundary of $P$. This means that we do not really need to compute the entire overlay $\omega$-only the intersections of $\omega$ with the boundary of $P$. These intersections can be computed in $O(n)$ time by computing the vertical and horizontal decompositions of $P$ separately. We call the set of intervals thus computed $\omega^{\prime}$. Once again, we are given the exits $e_{1}$ and $e_{2}$.

We create two pointers $i_{1}$ and $i_{2}$ with which we walk through the intervals in $\omega^{\prime}$. For each pair of intervals pointed to by $i_{1}$ and $i_{2}$ that we visit, we measure the number of squares that must be in the $e_{1}$ - and $e_{2}$-exit classes if we assume that the endpoints of their connections to the boundary of $P$ begin and end in $i_{1}$ and $i_{2}$. We call these areas $A_{1}$ and $A_{2}$ respectively. If we ever visit a pair of intervals for which $A_{1}$ and $A_{2}$ are both less than $A / 2$, then we divide the remaining squares so that both $A_{1}$ and $A_{2}$ are $A / 2$ - see Lemma 3 for detailsand return the results. Otherwise, we return the exit class that has size greater than $A / 2$, but whose size is minimal.

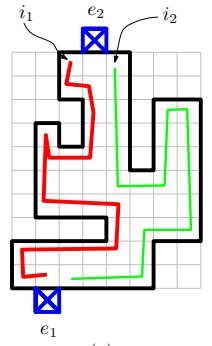

(a)

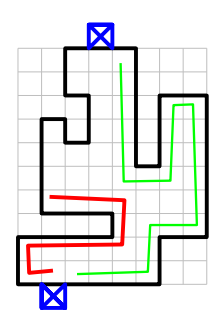

(b)

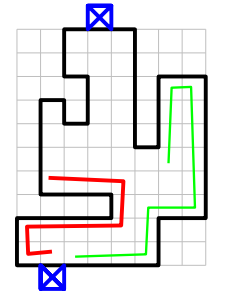

(c)

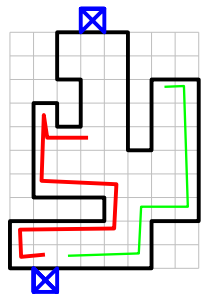

(d)

Fig. 5. An example of the linear-time algorithm. The pointers $i_{1}$ and $i_{2}$ denote the endpoints of the connection of the $e_{1}$-exit class to the boundary of the polygon. 
To begin with, we set $i_{1}$ and $i_{2}$ to be the interval containing $e_{2}$, so that the endpoints of the connection of the $e_{1}$-exit class are on either side of $e_{2}$. See Figure 5 (a). We call the area that the $e_{1}$-exit class must have $A_{1}$ and the area that the $e_{2}$-exit class must have $A_{2}$. We then move $i_{1}$ closer to $e_{1}$ until it either reaches $e_{1}$ or would cause $A_{2}$ to be greater than $A / 2$. As we progress, we simply update $A_{1}$ and $A_{2}$ and keep track of the smallest value for $A_{1}$. See Figure 5 (b).

Once we have done this for $i_{1}$, we do the same for $i_{2}$. See Figure 5 (c). Finally, we move $i_{1}$ back towards $e_{2}$. For each interval that we move $i_{1}$ towards $e_{2}$, we move $i_{2}$ as much as possible towards $e_{1}$ so that $A_{1}$ is as small as possible without causing $A_{2}$ to be larger than $A / 2$. See Figure 5 (d). As before, we keep track of the smallest value for $A_{1}$. When $i_{1}$ reaches $e_{2}$ or $i_{2}$ reaches $e_{1}$, we stop.

When we have completed the algorithm for $e_{1}$, we repeat the process, switching $e_{1}$ and $e_{2}$.

Theorem 3. In the confluent setting, the above algorithm finds the optimal evacuation plan for a polygon $P$ with two exits in $O(n)$ time, where $n$ is the number of vertices in $P$.

Proof. It is easy to see that this algorithm runs in linear time since each time we move a pointer, we only need to update the area currently assigned to the $e_{1}$-class. This takes time proportional to the complexity of the area that we are adding or removing, and the sum of the complexities of the updated areas is at most $2 n$.

We must argue, however, that the algorithm finds the minimum value for $A_{1}$ if $A_{1}$ must be greater than $A / 2$ in the optimal layout (a symmetric argument holds for $A_{2}$ ). The first two phases of the algorithm simply reduce $A_{1}$ as much as possible while keeping $i_{2}$ as close to $e_{2}$ as possible. The third phase is similar to the rotating calipers algorithm [15]. An invariant is established-in this case, $A_{1}$ is as small as possible for the given interval $i_{2}$ without making $A_{2}$ be greater than $A / 2$ - and then two pointers are moved in the same direction around $P$ while maintaining the invariant. In our algorithm $i_{2}$ visits every reasonable interval between $e_{1}$ and $e_{2}$, meaning that we eventually find the optimum interval.

\subsection{Dividing the interior}

As noted in the algorithms in Section 2.2, we need a subroutine that assigns grid squares to exits once the grid squares along the boundary of $P$ have been assigned. Given a connected subpolygon $P^{\prime} \subseteq P$, a natural number $x$, and an initial connected set $E_{i} \subseteq P^{\prime}$ of squares that belong to the exit class $e$ we wish to find a set $S$ of squares, where $|S|=x$ (the size condition) and where both $S \cup E_{i}$ and $P-\left(S \cup E_{i}\right)$ are connected (the connectivity condition). More precisely, since $x$ could be exponential in the complexity of the input, we would like to find a polygon that has complexity linear in the input that represents the boundaries of $S$.

We solve this problem for the useful case in which $P^{\prime}$ is assumed to have no boundary squares except those that are in $E_{i}$. In this case, we do not need to 


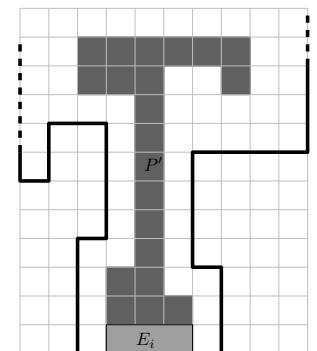

(a)

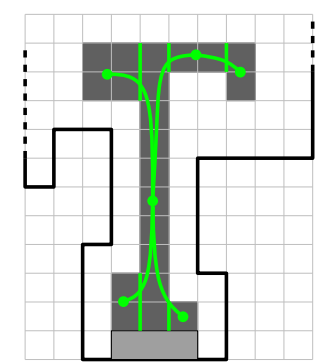

(b)

Fig. 6. (a) $P^{\prime}$ and $E_{i}$. (b) The vertical decomposition and its dual.

worry about the connectivity condition as long as $S \cup E_{i}$ is a connected polygon without holes. This is because any square in $P-\left(S \cup E_{i}\right)$ has a path to the boundary. Since $E_{i}$ is a connected set, the boundary squares of $P-\left(S \cup E_{i}\right)$ are a connected subset of $P$.

It is thus relatively simple to find $S$ given the assumption that $P^{\prime}$ may not contain any boundary squares of $P$. First, we check whether $\left|P^{\prime}\right|>x$. If it is not, we simply return the whole of $P^{\prime} \cup E_{i}$. Otherwise, we find the vertical decomposition of $P^{\prime} \cup E_{i}$. We perform a breadth-first-traversal of the dual of this vertical decomposition starting at a rectangle that contains a square of $E_{i}$, and adding the squares from each visited rectangle to $S$ as we go. We stop the traversal when $|S|+\left|E_{i}\right| \geq x$. We subtract the appropriate amount of squares from $S$ and return it.

Lemma 3. The above algorithm runs in $O(n)$ time and has output size that is linear in the input size.

Proof. Finding a vertical decomposition of a polygon can be completed in linear time [4]. Since the area of each rectangle of the decomposition can be computed in constant time, the time required for the breadth-first-traversal of the dual of the decomposition is also $O(n)$. Finally, we note that each rectangle that we add during the traversal can be charged to one of the vertices of the input, proving the second part of the lemma.

We conjecture that one can use algorithms similar to the naïve algorithm given above to compute the evacuation of any polygon with a constant number of exits, but the details become much more involved. We therefore leave this question to future work.

\section{Non-Confluent Flows}

Compared with confluent flows, non-confluent flows are clearly a stronger model. We note that any confluent flow is a non-confluent flow, but not vice versa. We show that non-confluent flows can be as much as $2-2 /(k+1)$ times as fast as 
confluent flows by giving an example in which this is the case. We then argue that the ratio our example achieves is optimal.

\subsection{Pseudo-Polynomial Algorithm}

In contrast to the case with confluent flows, for which we showed that finding an assignment of people to exits is strongly NP-complete when we are dealing with polygons with holes and $O(n)$ exits, we can show that, for non-confluent flows, a pseudo-polynomial algorithm exists.

The algorithm is based on the technique of using time-expanded networks to compute flows over time [13]. Therefore, we compute a flow network from the input polygon as follows. We create a source vertex $s$ and a sink vertex $t$. For each grid square in $P$, we create two vertices - an in vertex and an out vertex. We connect the in vertex to the out vertex with an edge that has capacity 1 for every grid square. We then make, for some integer $T \geq 1, T$ copies of the polygon $P_{1}, \ldots, P_{T}$, where each copy has these vertices and edges added. For every grid square of $P_{1}$, we connect $s$ to the in vertex of the grid square with an edge that has capacity 1 . We then connect the out vertex of every grid square in $P_{i}$ to the in vertex of all its neighbors in $P_{i+1}$ for all $1 \leq i \leq T-1$. Again, the edges we use all have capacity 1 . Finally, we connect the out vertex of every exit to $t$ with an edge that has capacity 1 . We call this flow network $G$.

It is fairly easy to see that if we are able to find a maximum flow of value $A$ through $G$, then we are able to evacuate $P$ in $T$ time steps. However, we note that both $T$ and $|G|$ can be exponential in the complexity of $P$, making this a pseudo-polynomial algorithm.

Theorem 4. There exists a pseudo-polynomial algorithm to find an evacuation of a polygon with a non-confluent flow.

\subsection{Differences to Confluent Flows}

The example that shows a large gap between confluent and non-confluent flows is a horizontal rectangle of width 1 with length $2 k+m k$, for some integer $m \geq 1$. Attached to this rectangle are $k$ vertical rectangles of width 1 and length $m k-$ one at every other square for the first $2 k$ squares. Between each vertical rectangle is an exit. Each exit can only be entered from the square to the left. See Figure $7(\mathrm{a})$.

We can see that the example has an optimal confluent flow that requires $2 m k+3$ time steps: three to remove the people directly to the left of each exit and all the people below the exits, $m k$ to remove the people in the vertical rectangles, and another $m k$ for the people in the horizontal "tail" to go through the rightmost exit. On the other hand, in the optimal non-confluent flow, all exits can remain continuously busy. One way that this can happen is for $m$ people from the horizontal rectangle to leave through successive exits, while people from the vertical rectangles are leaving through the other exits. Since the exits are continuously busy, the amount of time for all people to leave is 


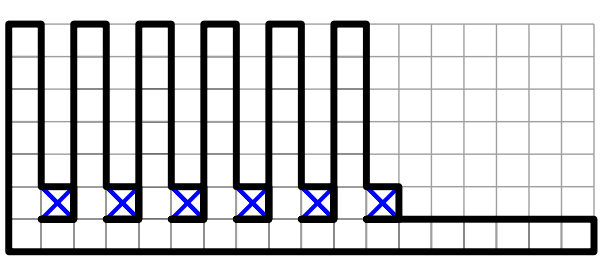

(a)

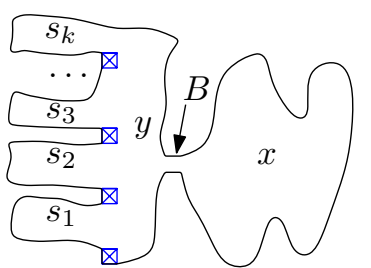

(b)

Fig. 7. (a) A polygon whose optimal non-confluent flow is nearly twice as fast as its optimal confluent flow. (b) The general shape of any polygon that realizes the maximum ratio between the confluent and non-confluent flows.

$\left(k^{2} m+(2+m) k\right) / k=m k+2+m$. The ratio between the confluent and nonconfluent flows in this case is

$$
\frac{2 m k+3}{m k+m+2}=2-\frac{2 m+1}{m k+m+2} \underset{m \rightarrow \infty}{\rightarrow} 2-\frac{2}{k+1} .
$$

We now show that the ratio achieved in this example is tight. Our ratio is similar to (and inspired by) the upper bound for the list-scheduling approximation ratio [6] in machine scheduling.

Theorem 5. The maximum ratio between the confluent flow and non-confluent flow in any grid polygon $P$ is $2-(2 /(k+1))$.

Proof. Let the ratio between the confluent flow and the non-confluent flow for a given polygon $P$ be known as $R_{P}$. When calculating $R_{P}$, we assume that the confluent and non-confluent flows are calculated optimally for $P$.

We begin by observing that by reducing the size of the smallest bottleneck in a polygon $P$ can only increase $R_{P}$. Suppose we have polygons $P_{a}$ and $P_{b}$, where $P_{a}$ has a minimum bottleneck size of at least 2 , and $P_{b}$ is the same as $P_{a}$, except that one grid square has been removed from the minimum bottleneck. The number of exit classes on one side of the smallest bottleneck in the confluent case can only decrease in $P_{b}$ relative to $P_{a}$, while in the non-confluent case, they may stay the same. Thus, it is possible that there exists an exit in the nonconfluent solution that is used for longer than in the confluent solution. This implies that the number of steps that is required to evacuate the building in the confluent setting increases faster than the number of steps required under the non-confluent setting. Therefore, $R_{P_{a}}$ is at least as large as $R_{P_{b}}$, and might be larger.

This implies that $R_{P}$ is maximized when the size of the minimum bottleneck is minimized, so we can assume that the size of the minimum bottleneck is 1 . We call the subpolygons on either side of the bottleneck $P_{1}$ and $P_{2}$.

Furthermore, we can easily see that increasing the difference between the number of exits in $P_{1}$ and $P_{2}$ can only increase $R_{P}$. This is because the number of people that must go through the bottleneck that separates $P_{1}$ and $P_{2}$ can only be increased by increasing this difference. Therefore, we can assume that 
all the exits are on one side of the bottleneck between $P_{1}$ and $P_{2}$. Without loss of generality, assume that all exits are in $P_{1}$.

Given this setup, we attempt to construct $P$ so that as many exits as possible are used during as many time steps as possible in the non-confluent case. This implies that there must be some source of people in $P_{1}$. This is because only one person can go through the bottleneck between $P_{1}$ and $P_{2}$ per time step. Therefore, at most one person from $P_{2}$ can reach an exit per time step. However, by creating a supply of people in $P_{1}$, we allow the people from $P_{2}$ to queue in front of the exits.

So that the people from $P_{2}$ can queue in front of the exits, the route taken by the people in $P_{1}$ from the supply to the exit must not interfere with the paths of the people from $P_{2}$ to the exits. This means that the number of people in $P_{1}$ must be split and distributed to each exit.

Therefore, $P$ has the form sketched in Figure $7(\mathrm{~b})$. There is a bottleneck $B$. The number of people behind $B$ is $x$, the amount of space for these people to queue in is $y$, and the supply of people for each exit $e_{i}$ is $s_{i}$.

In both the confluent and non-confluent solutions, it takes time $2 y / k$ to remove the $y$ people in the queueing area. In the non-confluent solution, it is necessary that this is the first step performed. After this is done, the people from behind the bottleneck begin entering the queueing area. The people must therefore take turns exiting from the queueing area and exiting from the supplies that are attached to each exit. This implies that $y$ is as small as possible (while satisfying $y \geq 2 k$ ) and that $s_{i} \geq x$ for all $1 \leq i \leq k$. Having different values of $s_{i}$ provides no advantage, so we assume that the value of $s_{i}$ is some value $s_{x}$ for all $i$.

The ratio between the confluent flow and non-confluent flow is thus

$$
\frac{2 y / k+2 s_{x}+2 x}{2 y / k+2 s_{x}+2 x / k}
$$

which is maximized according to our constraints when $s_{x}=x$ and when $y=2 k$. This gives a ratio of

$$
\frac{2 x+2 k / k}{x+x / k+2 k / k}=\frac{2 x+2}{x+x / k+2}=2-\frac{2 x+2 k}{x k+x+2 k} \underset{x \rightarrow \infty}{\rightarrow} 2-\frac{2}{k+1}
$$

which is the claimed result.

\section{Conclusions}

We have discussed evacuations in grid polygons. We first showed that finding evacuations with confluent flows in polygons with holes is hard, even for polygons with only two exits. We then looked at algorithms to find evacuations with confluent flows. Finally, we showed that, while the difference between confluent and non-confluent flows is potentially significant, it is bounded.

Our work raises some questions that require further study. For simple polygons, there is evidence that a constant number of exits allows strongly polynomial 
solutions, even though some of the technical details are complicated. What is the complexity of finding an evacuation plan with a confluent flow when the number of exits is not constant? Next, can we find an fixed-parameter-tractable algorithm to find the confluent evacuation of polygons? Finally, can we find a polynomial algorithm that gives the optimal evacuation using non-confluent flows? Note that it is not even clear that the output size of such an algorithm is always polynomial.

\section{Acknowledgments}

We thank Estie Arkin, Michael Bender, Joe Mitchell, and Martin Skutella for helpful discussions; Martin Skutella is also part of ADVEST.

\section{References}

1. H. Bast and S. Hert. The area partitioning problem. In Proc. 12th Can. Conf. Comput. Geom. (CCCG '00), pages 163-171, Fredericton, NB, Canada, 2000.

2. N. Baumann and M. Skutella. Earliest arrival flows with multiple sources. Math. Oper. Res., 34(2):499-512, 2009. Journal version of 2006 FOCS article "Solving evacuation problems efficiently".

3. R. Becker, I. Lari, M. Lucertini, and B. Simeone. Max-min partitioning of grid graphs into connected components. Networks, 32(2):115-125, 1998.

4. B. Chazelle. Triangulating a simple polygon in linear time. Discrete and Computational Geometry, 6(5):485 - 524, 1991.

5. M. R. Garey and D. S. Johnson. Computers and Intractability: A Guide to the Theory of NP-Completeness. W. H. Freeman, 1979.

6. R. L. Graham. Bounds on multiprocessing timing anomalies. SIAM Journal on Applied Mathematics, 17:416-429, 1969.

7. E. Györi. On division of graphs to connected subgraphs. In Combinatorics, pages 485-494, Keszthely, 1978.

8. L. Lovász. A homology theory for spanning trees of a graph. Acta Mathematica Hungarica, 30(3):241-251, 1977.

9. V. Lumelsky. Polygon area decomposition for multiple-robot workspace division. Int. Journal of Computational Geometry and Applications, 8(4):437-466, 1998.

10. J. Ma and S. Ma. An $O\left(k^{2} n^{2}\right)$ algorithm to find a $k$-partition in a $k$-connected graph. Journal of Computer Science and Technology, 9(1):86-91, 1994.

11. C. Moore and J. Robson. Hard tiling problems with simple tiles. Discrete and Computational Geometry, 26(4):573-590, Dec. 2001.

12. L. R. Salgado and Y. Wakabayashi. Approximation results on balanced connected partitions of graphs. Electr. Notes in Discrete Mathematics, 18:207-212, Dec. 2004.

13. M. Skutella. An introduction to network flows over time. In Research Trends in Combinatorial Optimization, pages 451-482. Springer-Verlag, 2009.

14. H. Suzuki, N. Takahashi, and T. Nishizeki. A linear algorithm for bipartition of biconnected graphs. Information Processing Letters, 33(5):227-231, 1990.

15. G. Toussaint. Solving geometric problems with the rotating calipers. In Proc. IEEE MELECON '83, pages 10-02, 1983. 